\title{
CATÁLOGO DE ALGAS MARINHAS BENTÔNICAS DO ESTADO DA BAHIA, BRASIL.
}

\author{
José Marcos de Castro NUNES
}

RESUMO. Catálogo de algas marinhas bentônicas do Estado da Bahia, Brasil. Totalizaram-se 258 táxons infragenéricos distribuídos em 145 Rhodophyta, 46 Phaeophyta e 67 Chlorophyta. Foram acrescidos ainda 35 novas citações para o litoral baiano.

Palavras chave. algas marinhas bentônicas, catálogo, Bahia, Brasil.

ABSTRACT. Check-list of seaweeds from Bahia State, Brazil. A checklist of 258 infrageneric taxa were listed of Bahia State, Brazil. This list comprises 145 species of Rhodophyta, 46 Phaeophyta and 67 Chlorophyta. 35 taxa were recorded in the first time for Bahia State.

Key words. seaweeds, check-list, Bahia, Brazil.

\section{INTRODUÇÃO}

Os primeiros registros sobre algas marinhas bentônicas no Estado da Bahia datam do século passado, podendo citar como exemplo: Martius et al. (1833), Martens (1870), Dickie (1874), Piccone (1885, 1886a, 1886b e 1889) De Toni (1889), Möbius (1889, 1890), e Weber van Bosse (1898), onde publicam listas de espécies ou descrições incompletas a partir de material proveniente de excursões, cujos dados sobre os locais de coleta eram pouco precisos. No início deste século surgiram outros como Gepp \& Gepp (1905), Howe (1928), Taylor (1930, 1931), Howe e Taylor (1931), e Williams \& Blomquist (1947) Chou (1954).

A partir da década de sessenta começam a aparecer os trabalhos de Aylton Brandão Joly e colaboradores sobre adições a flora brasileira, mencionando alguns táxons para o Estado da Bahia (Joly et al. 1965a, 1965b, 1967, 1969a, 1969b, 1976, Joly \& Ugadim 1966).

Citação de trabalhos contendo referências de espécies coletadas neste litoral são encontradas em Yamaguishi-Tomita (1970), OliveiraFilho (1974, 1977), Pinheiro-Joventino (1977), Semir (1977), Oliveira-Filho et al. (1979), Gouvea \& Leite (1980), Leite (1982), Kanagawa (1984), Teixeira et al. (1985), Braga (1986), Ugadim (1987), Oliveira (1989), Moura (1994), Guimarães (1996) e Buys \& Széchy (1997).

O primeiro levantamento sistemático de algas marinhas bentônicas realizado para o Estado da Bahia foi publicado por Martins et al. (1991), que faz referência a 33 táxons de 
clorofíceas marinhas da orla oceânica de Salvador. Em seguida Santos (1992), realizou trabalho sobre a microdistribuição e composição das comunidades de macroalgas em recifes na Ilha de Itaparica, listando 43 táxons. Mais recentemente Altamirano \& Nunes (1997) e Nunes (1997a, 1997b) publicaram trabalhos referentes a material coletado nos municípios de Salvador e Camaçari.

Oliveira-Filho (1977) catalogou todas as algas marinhas citadas para o Brasil até aquele período, fornecendo sua distribuição para 0 litoral brasileiro e os autores que as estudaram, incluindo inclusive material coletado ou examinado pelo próprio autor.

Este catálogo pretende servir como ponto de partida para estudos de levantamentos taxonômicos realizados para o Estado da Bahia, reunindo e organizando os dados da literatura, e consequentemente facilitando a sua consulta. Parte destes trabalhos, ao menos os mais significativos a exemplo de Oliveira-Filho (1977) e Santos (1992), acham-se sob forma de Tese ou Dissertação respectivamente, dificultando por conseguinte sua localização e consulta.

Alguns táxons foram acrescidos como primeira referência para o litoral baiano e litoral nordestino.

Exclui-se deste Catálogo as Divisões Cyanophyta e Chrysophyta.

\section{MATERIAL E MÉTODOS}

São fornecidos o nome do táxon, citação do autor e ano de publicação, em ordem cronológica. Dentro do parêntese ao lado do ano de publicação aparece o nome do táxon referido originalmente pelo autor.

Este catálogo teve como base OliveiraFilho (1977), acrescido por publicações posteriores.

Os táxons que constituem novas citações para o litoral baiano e/ou nordestino, vêm acompanhados de informações sobre município e praia onde foram coletados, número de Herbário, habitat e por vezes, fase reprodutiva e associações com outras algas e estáo assinalados por um asterisco. Todo material encontra-se registrado no Herbário Alexandre Leal Costa (ALCB) do Instituto de Biologia da Universidade Federal da Bahia.

A grande maioria dos táxons sem asterisco foram reconfirmados em coletas atuais.

Oliveira-Filho (1977) menciona alguns táxons como sendo "espécies duvidosas", por considerar de validez discutível ou de ocorrência improvável, estas então incluídas ao final do artigo.

Os táxons de categoria igual ou superior a família foram dispostos segundo sistema adotado por Wynne (1998) e Silva et al. (1987, 1996). Os táxons de categoria infragenérica são citados de forma mais atualizada possível, seguindo os trabalhos citados anteriormente. Dentro das famílias, os gêneros, e nestes as espécies, estão dispostos em ordem alfabética.

\section{RESULTADOS}

Foram listados 258 táxons infragenéricos, sendo 145 Rhodophyta distribuídos em 14 ordens e 25 famílias, 46 Phaeophyta com 08 ordens e 10 famílias, 67 Chlorophyta com 04 ordens e 14 famílias. Estão incluídas 35 novas citações para o litoral baiano, e 15 para 0 litoral nordestino ampliando assim a distribuição de algumas espécies para o litoral brasileiro.

Em seguida apresentamos a lista dos táxons.

\section{RHODOPHYTA}

\section{BANGIOPHYCIDAE}

\section{PORPHYRIDIALES}

Porphyridiaceae

Stylonema alsidii (Zanardini) Drew Joly, et al. (1969a como Goniotrichum alsidii). 


\section{COMPSOPOGONALES}

Erythrotrichiaceae

Erythrotrichia carnea (Dillwyn) J. Agardh

Oliveira-Filho et al. (1979) e Teixeira et al. (1985).

\section{Erythrotrichia porphyroides Gardner*}

Bahia: Salvador, Praia de Stella Maris (ALCB 22500); Conde, Barra do Itariri (ALCB 31530); Camaçari Praia de Jauá, (ALCB 31506). Coletada no mesolitoral. Epifitando Padina pavonica e $P$. gymnospora.

Primeira referência para o litoral nordestino.

Sahlingia subintegra (Rosevinge) Kornmann

Teixeira et al. (1985).

\section{BANGIALES}

Bangiaceae

Porphyra acanthophora Oliveira \& Coll*

Bahia: Uruçuca, Praia de Serra Grande (ALCB 32305). Coletada no mesolitoral superior.

\section{FLORIDEOPHYCIDAE}

\section{ACROCHAETIALES}

Acrochaetiaceae

Acrochaetium hallandicum (Kylin) Hamel

Oliveira-Filho et al. (1979 como Acrochaetium sargassi).

Acrochaetium microspicum (Nägeli ex Kützing) Nägeli

Bahia: Conde, Barra do Itariri (ALCB 32258). Coletada no mesolitoral. Epifitando Padina gymnospora.

\section{PALMARIALES}

Rhodothamniellaceae

Rhodothamniella codicola (Borgesen) Bidoux \& F. Magne Teixeira et al. (1985 como Acrochaetium codicola).

\section{CORALLINALES}

\section{Corallinaceae}

Amphiroa anastomosans Weber-van Bosse

Martins et al. (1991), Santos (1992), Altamirano \& Nunes (1997) e Nunes (1997b).

Amphiroa beauvoisii Lamouroux

Martinsetal.(1991), Santos(1992) e Nunes(1997b).

Amphiroa brasiliana Decaisne

Howe (1928), Taylor (1931), Santos (1992) e Nunes (1997b).

Amphiroa fragilissima (Linnaeus) Lamouroux

Martins et al. (1991), Oliveira-Filho (1977), Santos (1992) e Nunes (1997b).

Amphiroa rigida Lamouroux Santos (1992).

Corallina officinalis Linnaeus Martens (1870) e Taylor (1931).

Corallina panizzoi Schnetter \& Richter Moura (1994) e Buys \& Széchy (1996).

Haliptilon subulatum (Ellis \& Solander) Johansen Piccone (1886b como Corallina subulata), Martens (1870 como Corallina subulata), Howe (1928 como Corallina subulata), Taylor (1931 como Corallina subulata), Moura (1994) e Nunes (1997a, 1997b).

\section{Jania adhaerens Lamouroux}

Martins et al. (1991) e Nunes (1997a, 1997b).

Jania capillaceae Harvey

Howe (1928), Taylor (1931) e Oliveira-Filho et al. (1979).

Jania pumila Lamouroux

Joly et al. (1969a) e Nunes (1997b).

Jania rubens (Linnaeus) Lamouroux Taylor (1931).

Lithoporella atlantica (Foslie) Foslie

Oliveira-Filho et al. (1979 como Fosliella atlantica). 
Melobesia membranacea (Esper) Lamouroux

Taylor (1931 como Lithothamnion membranaceum).

\section{Neogoniolithon mamillare (Harvey) Setchell \& Mason \\ Martens (1870 como Melobesia mamillaris) e Taylor (1931 como Goniolithon mamillare).}

\section{GELIDIALES}

Gelidiaceae

\section{Gelidium coarctatum Kützing}

Ugadim (1987) e Altamirano \& Nunes (1997).

\section{Gelidium pusillum (Stackhouse) Le Jolis}

Ugadim (1987 como G. crinale), Santos (1992 como Gelidium pusillum var. pusillum) e Nunes (1997b).

\section{Gelidium spinulosum (Gmelin) Silva}

Taylor (1931 como Gelidium corneum).

\section{Pterocladia bartleethii Taylor*}

Bahia: Lauro de Freitas, Praia de Villas do Atlântico (ALCB 32276). Crescendo no mesolitoral. Plantas tetraspóricas.

Pterocladiella caerulescens (Kützing) Santelices \& Hommersand

Ugadim (1987 como Pterocladia caerulescens), Santos (1992 como G. pusillum var. conchicola), Altamirano \& Nunes (1997 como $P$. caerulescens) e Nunes (1997b como $P$. caerulescens).

\section{Gelidiellaceae}

Gelidiella acerosa (Forsskål) J. Feldmann \& Hamel Joly et al. (1969a), Martins et al. (1991), Santos (1992), Altamirano \& Nunes (1997) e Nunes (1997a, 1997b).

\section{NEMALIALES}

Liagoraceae

Ganonema farinosum (Lamouroux) Fan \& Wang* Bahia: Camaçari, Praia de Arembepe (ALCB
22171). Crescendo no infralitoral.

Primeira referência para o litoral nordestino.

Liagora ceranoides Lamouroux*

Bahia: Salvador, Praia de Itapoã (ALCB 32277). Crescendo no infralitoral.

Galaxauraceae

Galaxaura comans Kjelmann Joly et al. (1967).

Galaxaura marginata (Ellis \& Solander) Lamouroux

Martens (1870 como G. canaliculata), Taylor (1931), Chou (1945 como Galaxaura angustifrons), Altamirano \& Nunes (1997) e Nunes (1997b).

Galaxaura obtusata (Ellis \& Solander) Lamouroux Martens (1870), Howe (1928), Taylor (1931), Joly et al. (1969a também como Galaxaura breviarticulata), Teixeira et al. (1985) e Nunes (1997b).

Galaxaura rugosa (Ellis \& Solander) Lamouroux Martens (1870), Taylor (1931) e Joly, et al. (1969a como G. squalida).

Scinaia furcellata (Turner) J. Agardh*

Bahia: Salvador, Praia da Pituba (ALCB 32278); Camaçari, Arembepe (ALCB 22157); Praia de Jauá (ALCB 17214). Crescendo no infralitoral.

Tricleocarpa cylindrica (Ellis \& Solander) Huisman \& Borowitzka

Joly et al. (1969a como Galaxaura cylindrica).

Tricleocarpa fragilis (Linnaeus) Huisman \& Towns Taylor (1931 como Galaxaura oblongata), Santos (1992 como G. oblongata) e Altamirano \& Nunes (1997 como G. oblongata).

\section{BONNEMAISONIALES}

Bonnemaisoniaceae

Asparagopsis taxiformis (Delile) Trevisan

Joly et al. (1969a como Falkenbergia hillebrandii) e Teixeira et al. (1985 como Falkenbergia hillebrandii). 


\section{GIGARTINALES}

Corynomorphaceae

Corynomorpha clavata (Harvey) J. Agardh Nunes (1997b).

\section{Gigartinaceae}

Chondracanthus acicularis (Roth) Fredericq Altamirano \& Nunes (1997 como Gigartina acicularis)

\section{Chondracanthus teedii (Roth) Kützing}

Braga (1986 como Gigartina teedii), OliveiraFilho (1977 como Gigartina teedii) e Nunes (1997b como Gigartina teedii).

\section{Hypneaceae}

Hypnea cervicornis J. Agardh 1997b).

Altamirano \& Nunes (1997) e Nunes (1997a,

Hypnea musciformis (Wulfen in Jacquin) Lamouroux

Martius et al. (1833 como Sphaerococcus musciformis Wulfen), Howe (1928), Taylor (1931), Martins et al. (1991), Santos (1992), Altamirano \& Nunes (1997) e Nunes (1997a, 1997b).

Hypnea spinella (C. Agardh) Kützing

Martens (1870 como H. rissoana), Howe (1928), Taylor (1931), Joly, et al. (1969a), Oliveira (1989), Altamirano \& Nunes (1997 também como Hypnea cervicornis) e Nunes (1997a, 1997b também como H. cervicornis).

\section{Peyssonneliaceae}

\section{Peyssonnelia inamoena Pilger*}

Bahia: Camaçari, Praia de Arembepe (ALCB 32278). Crescendo no infralitoral.

Primeira referência para o litoral nordestino.

\section{Rhizophyllidaceae}

Ochtodes secundiramea (Montagne) Howe

Joly e Ugadim (1966), Joly et al. (1969a) e Nunes (1997b).
Solieriaceae

Meristiella gelidium (J. Agardh) Cheney et Gabrielson*

Guimarães (1996).

Solieria filiformis (Kützing) Gabrielson*

Bahia: Vera Cruz, Praia da Penha (ALCB 32280). Coletada no mesolitoral. Planta tetraspórica.

\section{HALYMENIALES}

Halymeniaceae

Cryptonemia crenulata (J. Agardh) J. Agardh Martens (1870 como Phyllophora crenulata), Dickie (1874), Oliveira-Filho (1974), PinheiroJoventino (1977) e Altamirano \& Nunes (1997).

Cryptonemia seminervis (C. Agardh) J. Agardh

Pinheiro-Joventino (1977 como Cryptonemia luxurians), Teixeira et al. (1985 como C. luxurians), Altamirano \& Nunes (1997 como C. luxurians) e Nunes (1997b como C. luxurians).

Grateloupia filicina (Lamouroux) C. Agardh*

Bahia: Uruçuca, Praia de Serra Grande (ALCB 22270). Coletada no mesolitoral.

Halymenia duchassaingii (J. Agardh) Kylin Joly et al. (1965a).

Halymenia floresia (Clemente) C. Agardh Dickie (1874), Taylor $(1930,1931)$ e OliveiraFilho (1974).

\section{GRACILARIALES}

\section{Gracilariaceae}

Gracilaria caudata J. Agardh

Taylor (1930, 1931 como G. confervoides) e Altamirano \& Nunes (1997 como Gracilaria verrucosa).

Gracilaria cervicornis (Turner) J. Agardh

Taylor (1931), Altamirano \& Nunes (1997 também como G. ferox) e Nunes (1997b).

Gracilaria dominguensis Sonder ex Kützing 
Altamirano \& Nunes (1997).

Gracilaria lacinulata (West in Vahl) Howe (1931).

Dickie (1874 como G. multipartita) e Taylor

Gracilaria mammilaris (Montagne) Howe

Taylor (1930, 1931) e Nunes (1997b).

Gracilaria tepocensis (Dawson) Dawson

Altamirano \& Nunes (1997).

Hydropuntia cornea (J. Agardh) Wynne*

Bahia: Uruçuca, Praia de Serra Grande (ALCB 22252); Vera Cruz, Barra Grande (ALCB 22079). Coletada no mesolitoral.

\section{RHODYMENIALES}

\section{Champiaceae}

\section{Champia feldmannii Diaz-Piferrer}

Altamirano \& Nunes (1997) e Nunes (1997b).

Champia parvula (C. Agardh) Harvey*

Bahia: Vera Cruz, Barra do Pote (ALCB 32281 ).

Coletada no infralitoral. Plantas tetraspóricas.

Champia salicornioides Harvey*

Bahia: Mata de São João, Praia do Forte (ALCB 32282). Coletada no infralitoral.

Primeira citação para o litoral nordestino.

\section{Rhodymeniaceae}

Botryocladia occidentalis (Børgesen) Kylin

Martius et al. (1833 como Chondria ovalis var. uvaria), Martens (1870 como Gastroclonium uvaria), Taylor (1931 como Chrysymenia uvaria), Teixeira et al. (1985), Santos (1992), Altamirano \& Nunes (1997) e Nunes (1997a, 1997b).

Botryocladia pyriformis (Børgesen) Kylin Santos (1992) e Nunes (1997a, 1997b).

\section{Chrysymenia enteromorpha Harvey}

Taylor (1931).

Chrysymenia dickieana J. Agardh

Taylor (1931).
Coelarthrum cliftonii (Harvey) Kylin

Teixeira et al. (1985 como C. albertisii).

Gelidiopsis gracilis (Kützing) Vickers

Nunes (1997b).

\section{CERAMIALES}

Ceramiaceae

Aglaothamnion filiponei (Howe) Aponte*

Bahia: Uruçuca, Praia de Serra Grande (ALCB 32283). Coletada no mesolitoral. Plantas tetraspóricas.

Primeira referência para o litoral nordestino.

Aglaothamnion uruguayense (Taylor) Aponte \& Norris

Teixeira et al. (1985 Callithamnion uruguayense).

\section{Antithamnion antillarum $\mathrm{B} \emptyset \mathrm{rgesen}$}

Joly et al. (1969a).

Callithamnion corymbosum (J. E. Smith) Lyngbye Martius et al. (1833 como Ceramium versicolor) e Taylor (1931).

Centroceras clavulatum (C. Agardh in Kunth) Montagne in Durieu de Maisonneuve Joly et al. (1969a), Nunes (1997b).

\section{Centrocerocolax ubatubensis Joly*}

Bahia: Conde, Barra do Itariri (ALCB 32284).

Coletada no mesolitoral. Plantas tetraspóricas. Epifitando Centroceras clavulatum.

Primeira referência para o litoral nordestino.

\section{Ceramium brasiliense Joly}

Teixeira et al. (1985) e Altamirano \& Nunes(1997).

Ceramium comptum Børgesen

Teixeira et al. (1985).

Ceramium dawsonii Joly

Oliveira-Filho et al. (1979), Teixeira et al. (1985) e Altamirano \& Nunes (1997).

Ceramium luetzelburgii Schmidt

Oliveira-Filho et al. (1979). 
Ceramium tenerrimum (Martens) Okamura Joly et al. (1969a).

Crouania attenuata (C. Agardh) J. Agardh Joly et al. (1965a), Oliveira-Filho et al. (1979)

e Teixeira et al. (1985).

Diplothamnion tetrastichum Joly \& Yamaguishi Oliveira-Filho et al. (1979).

Dohrniella antillarum (Taylor) Feldmann-Mazoyer var. brasiliensis Joly \& Ugadim in Joly et al. Joly et al. (1969a) e Oliveira-Filho et al. (1979).

Griffithsia schousboei Montagne

Oliveira-Filho et al. (1979).

Griffithsia tenuis C. Agardh

Joly et al. (1969a).

Haloplegma duperreyi Montagne

Oliveira-Filho (1977).

Spermothamnion nonatoi Joly*

Bahia: Camaçari, Arembepe (ALCB 32285).

Coletada no infralitoral. Epizoica em hidrozoários.

Primeira referência para o litoral nordestino.

Spyridia clavata Kützing

Joly et al. (1969a).

Spyridia filamentosa (Wulfen) Harvey in Hooker Taylor (1930).

Spyridia hypnoides (Bory in Belanger) Papenfuss Nunes (1997b).

Tiffaniella gorgonea (Montagne) Doty \& Meñez

Joly et al. (1969a como Spermothamnion gorgoneum).

Wrangelia argus (Montagne) Montagne Joly et al. (1969a) e Nunes (1997a, 1997b).

\section{Dasyaceae}

Dasya baillouviana (S. G. Gmelin) Montagne

Teixeira et al. (1985).

Dictyurus occidentalis J. Agardh*
Bahia: Camaçari, Arembepe (ALCB 22163), Jauá (ALCB 17107). Coletadas no infralitoral.

Heterosiphonia crispella (C. Agardh) Wynne

Joly et al. (1969a como H. wurdemanni var. wurdemanii), Oliveira-Filho, Ugadim e Paula (1979 como H. wurdemanni var, laxa) e Teixeira et al. (1985 como H. wurdemanii).

\section{Delesseriaceae}

\section{Caloglossa leprieurii (Montagne) J. Agardh} Leite (1982) e Oliveira-Filho (1977).

\section{Claudea elegans Lamouroux*}

Bahia: Camaçari, Arembepe (ALCB 32286). Coletada no infralitoral. Oliveira-Filho (1977) faz referência deste táxon para a Bahia, mas na verdade, este foi dragado nos estados de Pernambuco e Rio de Janeiro (Joly et al. 1978).

Cottoniella filamentosa (Howe) Børgesen Joly et al. (1969a).

\section{Cryptopleura crispa Kylin}

Teixeira et al. (1985).

Hypoglossum tenuifolium (Harvey) J. Agardh Oliveira-Filho et al. (1979).

Nitophyllum wilkinsoniae Collins \& Hervey Joly et al. (1965a).

\section{Rhodomelaceae}

Acanthophora muscoides (Linnaeus) Bory

Martens (1870 como Chondria muscoides Agardh), Taylor (1931), Altamirano \& Nunes (1997) e Nunes (1997b).

Acanthophora spicifera (Vahl) Børgesen

Martius et al. (1833 como Chondria acanthophora), Martens (1870 como A. thierii), Taylor (1931), Oliveira (1989), Altamirano \& Nunes (1997) e Nunes (1997a, 1997b).

Amansia multifida Lamouroux

Piccone (1885, 1866a), Howe (1928), Taylor (1931) e Nunes (1997a, 1997b). 
Bostrychia calliptera (Montagne) Montagne Oliveira-Filho (1977).

\section{Bostrychia montagnei Harvey}

Joly et al. (1969a), Oliveira-Filho (1977 como

B. scorpioides var. montagnei) e Leite (1982).

Bostrychia radicans (Montagne) Montagne

Joly et al. (1969a).

Bostrychia tenella (Vahl) J. Agardh

Joly et al. (1969a como Bostrychia binderi), Leite (1982 como B. binderi) e Nunes (1997a, 1997b como B. binderi).

Bryocladia cuspidata (J. Agardh) De Toni*

Bahia: Conde, Sítio do Conde (ALCB 32287);

Barra do Itariri (ALCB 32297). Coletada no mesolitoral. Plantas tetraspóricas.

\section{Bryothamnion seaforthii (Turner) Kützing}

Dickie (1874), Howe (1928), Taylor (1930,

1931), Oliveira-Filho (1974), Martins et al. (1991) e Nunes (1997a, 1997b).

Bryothamnion triquetrum (S. G. Gmelin) Howe Howe (1928), Taylor (1931), Martins et al. (1991) e Nunes (1997a, 1997b).

\section{Chondria atropurpurea Harvey}

Dickie (1874, como Chondriopsis atropurpurea).

Chondria platymea Joly \& Ugadim in Joly et al. Oliveira-Filho et al. (1979).

\section{Chondria polyrhiza Collins \& Hervey}

Oliveira-Filho et al. (1979).

Digenea simplex (Wulfen) C. Agardh

Howe (1928), Taylor (1931), Martins et al. (1991), Santos (1992) e Nunes (1997a, 1997b).

Dipterosiphonia dendritica (C. Agardh) Schmitz in Engler \& Prantl

Taylor (1931) e Nunes (1997a, 1997b).

Enantiocladia duperreyi (C. Agardh) Falkenberg Nunes (1997a, 1997b).

Herposiphonia secunda (C. Agardh) Falkenberg
Joly et al. (1969a também como H. tenella) e Nunes (1997a, 1997b).

\section{Laurencia arbuscula Sonder*}

Bahia: Cairu, Ilha de Tinharé (ALCB 32288).

Coletada no mesolitoral. Plantas tetraspóricas.

Primeira referência para o litoral nordestino.

\section{Laurencia caraibica Silva}

Oliveira-Filho et al. (1979 como Laurencia nana).

Laurencia corallopsis (Montane) Howe*

Bahia: Camaçari, Guarajuba (ALCB 32289); Salvador, Praia de Stella Maris (ALCB 32290). Coletada no mesolitoral. Plantas tetraspóricas e masculinas. Associada a Anadyomene stellata e Dictyopteris delicatula.

Primeira referência para o litoral nordestino.

Laurencia flagellifera J. Agardh

Altamirano \& Nunes (1997).

Laurencia furcata Cordeiro-Marino \& Fujii*

Bahia: Salvador, Praia de Stella Maris (ALCB 32291); Lauro de Freitas, Praia de Villas do Atlântico (ALCB 32292). Coletada no mesolitoral. Plantas tetraspóricas e masculinas. Epifitadas por Hypnea musciformis e Herposiphonia secunda.

Primeira referência para o litoral nordestino.

\section{Laurencia intricata Lamouroux*}

Bahia: Cairú, Ilha de Tinharé (ALCB 32293).

Coletada no mesolitoral. Associada a Amphiroa fragilissima e Anadyomene stellata.

Primeira referência para o litoral nordestino.

Laurencia obtusa (Hudson) Lamouroux

Martius et al. (1833 como Chondria obtusa Agardh), Taylor (1931) e Joly et al. (1969a).

Laurencia oliveirana Yoneshigue*

Bahia: Conde, Barra do Itariri (ALCB 32297).

Coletada no mesolitoral. Associada a Bryocladia cuspidata e Amphiroa fragilissima.

Primeira referência para o litoral nordestino.

Laurencia papillosa (C. Agardh) Greville

Martius et al. (1833 como Chondria thyrsoidea), Martins et al. (1991), Santos (1992), 
Altamirano \& Nunes (1997) e Nunes (1997b).

Laurencia perforata (Bory) Montagne Santos (1992).

Laurencia translucida Fujii \& Cordeiro-Marino* Bahia: Camaçari, Praia de Arembepe (ALCB 32296); Salvador, Praia de Stella Maris (ALCB 32295); Uruçuca, Praia de Serra Grande (ALCB 32294). Coletada no mesolitoral. Plantas tetraspóricas, cistocárpicas e masculinas. Epifitadas por Dictyopteris delicatula e Ceramium dawsonii. Associada a Anadyomene stellata e Amphiroa anastomosans.

Primeira referência para o litoral nordestino.

Lophocladia trichocladus (C. Agardh) Schmitz

Joly et al. (1967) e Oliveira (1989).

Micropeuce mucronata (Harvey) Kylin

Taylor (1930, 1931 como Dasya sertularioides), Howe e Taylor (1931 como D. sertularioides).

Murrayella periclados (C. Agardh) Schmitz Oliveira-Filho (1977).

Osmundaria obtusiloba (C. Agardh) Norris Howe (1928 como Vidalia obtusiloba), Taylor (1931 como V. obtusiloba), Teixeira et al. (1985 como V. obtusiloba), Altamirano \& Nunes, (1997 como V. obtusiloba) e Nunes (1997a como V. obtusiloba).

Polysiphonia ferulacea Suhr ex J. Agardh Oliveira (1989).

Polysiphonia havanensis Montagne Joly et al. (1969a).

Polysiphonia subtilissima Montagne Oliveira-Filho et al. (1979).

Protokuetzingia schottii W. Taylor*

Bahia: Camaçari, Arembepe (ALCB 22165), Jauá (ALCB 17108). Coletada no infralitoral. Plantas tetraspóricas.

Pterosiphonia parasitica (Hudson) Falkenberg var. australis Joly \& Cordeiro-Marino

Joly et al. (1967b).
Wrightiella tumanowiczi (Gatty ex Harvey) Schmitz Dickie $\quad(1847 \mathrm{e}$ como Dasya tumanowiczi), Taylor (1931) e Oliveira-Filho (1974).

\section{PHAEOPHYTA}

\section{ECTOCARPALES}

Ectocarpaceae

Asteronema rhodochortonoides (Børgesen) D. Müller \& Parodi

Oliveira-Filho et al. (1979 como Ectocarpus rhodochortonoides).

\section{Bachelotia antillarum (Grunow) Gerloff*}

Bahia: Camaçari, Praia de Arembepe (ALCB 17065), Praia de Guarajuba (ALCB 32249), Praia de Itacimirim (ALCB 32248), Praia de Jauá (ALCB 32246); Salvador, Praia de Placafor (ALCB 17340), Praia de Itapoã (ALCB 31520), Praia de Stella Maris (ALCB 32241); Vera Cruz, Barra Grande (ALCB 32242); Conde, Barra do Itariri (ALCB 32247), Sítio do Conde (ALCB 31542); Uruçuca, Praia de Serra Grande (ALCB 22244). Coletada no mesolitoral. Associada a Hincksia breviarticulatus, Hincksia michelliae, Feldmania irregularis, Sphacelaria tribuloides, Blindgia marginata, Cladophora vagabunda e Asteronema rhodochortonoides.

Feldmannia irregularis (Kützing) Hamel Joly et al. (1969a como Ectocarpus irregulares).

Ectocarpus rallsiae (Vickers) W. Taylor Oliveira-Filho et al. (1979 Giffordia rallsiae).

Hincksia breviaticulata (J. Agardh) P. Silva Joly et al. (1969a como Ectocarpus breviarticulatus).

Hincksia mitchelliae (Harvey) P. C. Silva* Bahia: Camaçari, Praia de Arembepe (ALCB 32274); Salvador, Praia de Placafor (ALCB17333), Praia de Itapoã (ALCB 17351, 31522). Coletada no mesolitoral. Associada a Bachelotia antillarum. Epifitando Sargassum vulgare, Corallina panizzoi e Padina pavonica. 


\section{CHORDARIALES}

Elachistaceae

Elachista minutissima W. Taylor

Oliveira-Filho et al. (1979).

Ralfsiaceae

Ralfsia expansa (J. Agardh) J. Agardh*

Bahia: Camaçari, Praia de Jauá (ALCB 322300); Salvador, Praia de Itapoã (ALCB 32298), Praia do Farol da Barra (ALCB 32299). Coletada no mesolitoral.

\section{SPOROCHNALES}

Sporochnaceae

Sporochnus bolleanus Montagne

Taylor $(1930,1931)$.

\section{DICTYOSIPHONALES}

Punctariaceae

Hecatonema floridanum (W. Taylor) W. Taylor Joly et al. (1969a).

\section{SCYTOSIPHONALES}

\section{Chnoosporaceae}

Chnoospora minima (Hering) Papenfuss*

Bahia, Camaçari, Praia de Arembepe (ALCB 22343), Praia de Jauá (ALCB 22342); Conde, Sítio do Conde (ALCB 22408); Uruçuca, Serra Grande (ALCB 22276). Coletada no mesolitoral.

Scytosiphonaceae

Colpomenia sinuosa (Roth) Derbès \& Solier Joly et al. (1969a), Oliveira-Filho et al. (1979), Martins et al. (1991) e Nunes (1997a).

Hydroclathrus clathratus (C. Agardh) Howe Joly et al. (1969a).

Rosenvingea intricata (C. Agardh) B $\emptyset$ rgesen Martius (1833 como Ulva endiviaefolia),
Taylor (1931) e Joly et al. (1969a).

Rosenvingea sanctae-crucis Børgesen*

Bahia: Camaçari, Praia de Arembepe (ALCB 32301). Coletada no infralitoral.

\section{SPHACELARIALES}

Sphacelariaceae

\section{Sphacelaria rigidula Kützing}

Joly et al. (1969a como S. furcigera) e Oliveira-Filho et al. (1979 como S. furcigera).

Sphacelaria tribuloides Meneghini Joly et al. (1969a).

\section{DICTYOTALES}

Dictyotaceae

Dictyopteris delicatula Lamouroux

Martius et al. (1833 como Haliseris delicatula), Howe (1928 como Neurocarpus hauckianus), Taylor (1930 como Neurocarpus delicatulus, 1931 como $N$. delicatulus), Joly et al. (1969a), Teixeira et al. (1985), Oliveira (1989), Martins et al. (1991), Santos (1992), Altamirano \& Nunes (1997) e Nunes (1997a).

Dictyopteris justii Lamouroux

Martens (1870 como Haliseris justii), Taylor (1930, 1931 como Neurocarpus), Joly et al. (1969a) e Oliveira (1989).

Dictyopteris plagiogramma (Montagne) Vickers

Taylor (1931 como Neurocarpus plagiogrammus (Montagne) Kützing), Joly et al. (1969a), Teixeira et al. (1985).

Dictyopteris polypodioides (De Candolle) Lamouroux

Martius et al. (1833 como Haliseris polypodioides) e Altamirano \& Nunes (1997 como Dictyopteris membranaceae).

Dictyota bartayresiana Lamouroux

Oliveira (1989).

Dictyota cervicornis Kützing 
Joly et al. (1969a), Oliveira (1989) e Santos (1992).

\section{Dictyota ciliolata Kützing}

Joly et al. (1969a) e Oliveira (1989).

Dictyota jamaicensis W. Taylor

Joly et al. (1965a, 1969a) e Altamirano \& Nunes (1997).

Dictyota menstrualis (Hoyt) Schnnetter, Hörnig \& Weber-Peukert

Gepp \& Gepp (1905 como D. dichotoma), Taylor (1931 como D. dichotoma), Oliveira (1989 como D. dichotoma) e Altamirano \& Nunes (1997 como D. dichotoma).

\section{Dictyota mertensii (Martius) Kützing}

Martius et al. (1833 como Ulva mertensii), Martens (1870 como D. brongniartii), Howe (1928 como D. dentata e Dilophus guineensis), Taylor (1931 como D. dentata e Dilophus guineensis), Joly et al. (1969a como D. dentata), Oliveira-Filho et al. (1979) e Oliveira (1989).

Dictyota pulchella Hörniig \& Schnetter

Taylor (1930 como D. divaricata), Joly et al. (1969a como Dictyota linearis) e Oliveira (1989 como $D$. divaricata $\mathrm{ef}$.).

Lobophora variegata (Lamouroux) Womersley ex Oliveira

Martens (1870 como Gymnogongrus collaris, Padina fraseri e Zonaria collaris), Howe (1928 como Zonaria variegata), Taylor (1930 como Zonaria variegata, 1931 como Padina fraseri e Zonaria variegata), Joly et al. (1969a como Pocokiella variegata), Teixeira et al. (1985), Santos (1992) e Altamirano \& Nunes (1997).

Padina gymnospora (Kützing) Sonder

Joly et al. (1969a) e Altamirano \& Nunes (1997).

Padina pavonica (Linnaeus) Thivy in W. Taylor Taylor (1931).

Padina sanctae-crucis $\mathrm{B} \emptyset \mathrm{rgesen}$

Howe (1928) e Taylor $(1930,1931)$.

Padina tetrastromatica Hauck*
Bahia: Lauro de Freitas, Praia de Villas do Atlântico (ALCB 31552); Camaçari, Praia de Itacimirim (ALCB 22495), Praia de Jauá, (ALCB 224797). Coletadas no mesolitoral. Plantas esporofíticas.

Primeira referência para o litoral nordestino.

Spatoglossum schroederi (C. Agardh) Kützing Oliveira-Filho (1977) e Altamirano \& Nunes (1997).

Stypopodium zonale (Lamouroux) Papenfuss

Gepp \& Gepp (1905 como S. lobatum), Joly et al. (1969a) e Teixeira et al. (1985).

Zonaria tournefortii (Lamouroux) Montagne

Joly et al. (1969a) e Oliveira-Filho (1977).

\section{FUCALES}

\section{Sargassaceae}

Sargassum acinarium (Linnaeus) Setchell Joly et al. (1969a).

Sargassum cymosum C. Agardh

Martens (1870) e Taylor (1931).

Sargassum filipendula C. Agardh

Oliveira-Filho (1977).

\section{Sargassum furcatum Kützing*}

Bahia: Itaparica, Ponta de Areia (ALCB 32236). Coletada atirada à praia e em redes de pesca.

Primeira referência para o litoral nordestino.

Sargassum hystrix J. Agardh

Joly et al. (1969a), Oliveira-Filho et al. (1979) e Teixeira et al. (1985).

\section{Sargassum platycarpum Montagne}

Piccone (1886 como S. liebmanii), Taylor (1931 como S. liebmanii), Joly et al. (1969a) e Oliveira-Filho et al. (1979).

\section{Sargassum polyceratium Montagne}

Martens ( 1870 como S. bahiense Kg.), Piccone (1889 como S. spinulosum var. ciliata Grunow), Howe (1928) e Taylor (1931). 
Sargassum stenophyllum (Martens) Martius*

Bahia: Camaçari, Praia de Arembepe (ALCB 22462). Coletada no mesolitoral. Plantas masculinas.

\section{Sargassum vulgare C. Agardh}

Gepp \& Gepp (1905), Taylor (1931), Joly et al. (1969a) e Altamirano \& Nunes (1997).

\section{CHLOROPHYTA}

\section{ULVALES}

Gayraliaceae

Gayralia oxysperma Vinogradova ex Scagel et al. Oliveira-Filho (1977 como Ulvaria oxysperma).

\section{Monostromataceae}

Blidingia marginata (J. Agardh) P. Dangeard Altamirano \& Nunes (1997).

\section{Ulvaceae}

\section{Enteromorpha chaetomorphoides Børgesen}

Oliveira-Filho et al. (1979).

Enteromorphaflexuosa (Wulfen ex Roth) J. Agardh Taylor (1930), Martins et al. (1991) e Altamirano \& Nunes (1997).

\section{Enteromorpha lingulata J. Agardh Joly et al. (1969a).}

\section{Enteromorpha prolifera (Müller) J. Agardh} Howe (1928) e Taylor (1931).

Ulva fasciata Delile

Oliveira-Filho (1977), Kanagawa (1984), Martins et al. (1991), Altamirano \& Nunes (1997) e Nunes (1997a, 1997b).

\section{Ulva lactuca Linnaeus}

Martius et al. (1833), Howe (1928 também como U. rigida), Taylor (1931 também como $U$. lactuca f. rigida), Kanagawa (1984), Oliveira (1989), Martins et al. (1991), Altamirano \& Nunes (1997) e Nunes (1997a, 1997b).

\section{Ulvellaceae}

Bolbocoleon jolyi Yamaguishi-Tomita Yamaguishi-Tomita (1970).

\section{CLADOPHORALES}

Anadyomenaceae

Anadyomene stellata (Wulfen) C. Agardh

Howe (1928), Taylor (1930, 1931), Joly et al. (1969a), Teixeira et al. (1985), Martins et al. (1991), Santos (1992), Altamirano \& Nunes (1997) e Nunes (1997b).

\section{Microdictyon boergesenii Setchell} Taylor (1931).

\section{Microdictyon vanbosseae Setchell} Teixeira et al. (1985).

\section{Cladophoraceae}

\section{Chaetomorpha antennina (Bory) Kützing}

Howe (1928 como C. media), Taylor (1931 como C. media) e Martins et al. (1991).

Cladophora coelothrix Kützing Martins et al. (1991).

\section{Cladophora prolifera (Roth) Kützing} Martins et al. (1991) e Altamirano \& Nunes (1997).

Cladophora vagabunda (Linnaeus) van den Hoek* Bahia: Salvador, Praia da Pituba (ALCB 17434), Praia de Itapoã (ALCB 22122). Coletada no mesolitoral.

Rhizoclonium riparum (Roth) Kützing ex Harvey Joly et al. (1969a).

\section{Siphonocladaceae}

Chamaedoris peniculum (Solander) Kuntze Taylor (1930, 1931), Teixeira et al. (1985), Martins et al. (1991) e Nunes (1997a).

\footnotetext{
Cladophoropsis membranaceae (C. Agardh) Børgesen

Oliveira-Filho (1977) e Martins et al. (1991).
} 
Phyllodictyon anastomosans Kraft \& Wynne

Joly et al. (1965b como Struvea anastomosans)

e Martins et al. (1991 como S. anastomosans).

Ventricaria ventricosa (J. Agardh) Olsen \& J. West Taylor (1930, 1931 como Valonia ventricosa), Martins et al. (1991 como Valonia ventricosa).

Valoniaceae

Dictyosphaeria cavernosa (Forsskål) Børgesen Joly et al. (1969a).

Dictyosphaeria versluysii Weber-van Bosse Joly et al. (1969a, 1969b), Martins et al. (1991), Santos (1992), Altamirano \& Nunes (1997).

Valonia aegagropila C. Agardh

Joly et al. (1969a), Martins et al. (1991), Santos (1992) e Altamirano \& Nunes (1997).

Valonia macrophysa Kützing

Joly et al. (1969a), Martins et al. (1991).

\section{BRYOPSIDALES}

Chaetosiphonaceae

\section{Blastophysa rhizopus Reinke}

Teixeira et al. (1985).

\section{Bryopsidaceae}

Bryopsis pennata Lamouroux

Howe (1928 como B. harveyana), Taylor (1931 como B. pennata var. secunda), Martins et al. (1991), Altamirano \& Nunes (1997) e Nunes (1997b).

Bryopsis plumosa (Hudson) C. Agardh Joly et al. (1969a).

Derbesia marina (Lyngbye) Solier

Oliveira-Filho et al. (1979).

\section{Codiaceae}

Codium decorticatum (Woodward) Howe

Taylor (1931 como C. tomentosum) e Teixeira et al. (1985).
Codium intertextum Collins \& Harvey

Howe (1928), Taylor (1931), Martins et al. (1991) e Santos (1992).

\section{Codium isthmocladum Vickers}

Teixeira et al. (1985), Oliveira (1989) e Martins et al. (1991).

Codium repens P. \& H. Crouan ex Vickers Gepp \& Gepp (1905 como C. tomentosum).

\section{Codium taylorii Silva}

Martins et al. (1991).

\section{Caulerpaceae}

Caulerpa cupressoides (West in Vahl) C. Agardh

De Toni (1889 como Caulerpa lycopodium), Joly et al. (1969a), Martins et al. (1991 como C. cupressoides var. cupressoides e $C$. cupressoides var. lycopodium) e Altamirano \& Nunes (1997).

\section{Caulerpa fastigiata Montagne}

Howe (1928) e Martins et al. (1991).

\section{Caulerpa floridana $\mathrm{W}$. Taylor}

Joly et al. (1976).

\section{Caulerpa kempfii Joly \& Pereira*}

Bahia: Camaçari, Praia de Arembepe (ALCB 32302). Coletada no infralitoral.

Caulerpa lanuginosa J. Agardh

Joly et al. (1969a).

\section{Caulerpa mexicana Sonder ex Kützing}

Taylor (1930 como C. crassifolia f. mexicana), Taylor (1931 como C. crassifolia f. mexicana), Oliveira (1989) e Altamirano \& Nunes (1997).

Caulerpa prolifera (Forsskål) Lamouroux

Taylor (1931) e Joly et al. (1969a).

Caulerpa pusilla (Kützing) J. Agardh

Teixeira et al. (1985).

Caulerpa racemosa (Forsskål) J. Agardh var. peltata (Lamouroux) Eubank

Martens (1870 como Chauvinia chemnitzia), Taylor (1931 como C. peltata), Joly et al. (1969a 
como C. peltata), Martins et al. (1991) e Santos (1992).

Caulerpa racemosa (Forsskål) J. Agardh var. racemosa

Martius et al. (1833 como C. sedoides), Martens (1870 como C. sedoides), Taylor (1931 também como C. racemosa var. uvifera), Joly et al. (1969a), Martins et al. (1991), Santos (1992) e Altamirano \& Nunes (1997).

Caulerpa scalpelliformis (R. Brown ex Turner) C. Agardh

De Toni (1889 como C. denticulada), Joly et al. (1965a como C. scalpelliformis f. denticulata).

\section{Caulerpa serrulata (Forsskål) J. Agardh}

Martins et al. (1991).

Caulerpa sertularioides (S. G. Gmelin) Howe

Martins et al. (1991) e Altamirano \& Nunes (1997).

Caulerpa verticilata J. Agardh

Joly et al. (1969a), Teixeira et al. (1985) e Martins et al. (1991).

\section{Caulerpa webbiana Montagne}

Joly et al. (1969a).

Caullerpella ambigua (Okamura) Prud'homme \& Lokhorst

Joly et al. (1969a como Caulerpa vickersiae)

\section{Udoteaceae}

Avrainvillea nigricans Decaisne

Martins et al. (1991).

Boodleopsis pusilla (Collins) W. Taylor, Joly \& Bernatowicz*

Bahia: Conde. Barra do Itariri (ALCB 32303). Coletada no mesolitoral. Associada a Asteronema rhodochortonoides, Caulerpa fastigiata e Sphacelaria tribuloides.

\section{Halimeda discoidea Decaisne}

Joly et al. (1969a), Martins et al. (1991) e Altamirano \& Nunes (1997).
Halimeda incrassata (Ellis) Lamouroux Teixeira et al. (1985).

Halimeda opuntia (Linnaeus) Lamouroux

Martens (1870), Möbius (1889), Piccone (1889), Howe (1928), Taylor (1931), Gouvea \& Leite (1980), Martins et al. (1991), Santos (1992), Altamirano \& Nunes (1997) e Nunes (1997a, 1997b).

Halimeda tuna (Ellis \& Solander) Lamouroux Taylor $(1930,1931)$ e Joly et al. (1969a).

Penicillus capitatus Lamarck

Joly et al. (1969a) e Santos (1992).

Rhipilia fungiformis Joly \& Ugadim in Joly et al. Joly et al. (1965b).

Udotea cyathiformis Decaisne

Taylor (1930, 1931), Joly et al. (1969a) e Martins et al. (1991).

Udotea flabellum (Ellis \& Solander) Lamouroux Dickie (1874 como Udotea halimeda), Joly et al. (1969a), Oliveira-Filho (1974) e Martins et al. (1991).

Udotea occidentalis Gepp \& Gepp

Martens (1870 como U. halimeda Kützing) e Taylor (1931).

\section{DASYCLADALES}

Dasycladaceae

Neomeris annulata Dickie

Martins et al. (1991) e Santos (1992).

Dasycladus vermicularis (Scopoli) Krasser*

Bahia: Camaçari, Praia de Arembepe (ALCB 32304). Coletada no infralitoral.

$$
\text { Polyphysaceae }
$$

Acetabularia crenulata Lamouroux Joly et al. (1965a).

Acetabularia myriospora Joly \& Cordeiro-Marino Joly et al. (1965b), Martins et al. (1991 como Polyphysa myriospora) e Santos (1992 como Polyphysa myriospora). 
Acetabularia pusilla (Howe) Collins Santos (1992).

\section{ESPÉCIES DUVIDOSAS}

Lithothamnion fasciculatum (Lamarck) Areschoug Möbius (1889), Taylor (1931) e Oliveira-Filho (1977).

\section{Lithothamnion scabiosum (Harvey) Foslie} Taylor (1931) e Oliveira-Filho (1977).

\section{Sphaerococcus dumosus Martius}

Martius et al. (1833) e Oliveira-Filho (1977).

Gastroclonium ovatum (Hudson) Papenfuss

Martens (1870 como G. ovale), Taylor (1931

como G. ovale) e Oliveira-Filho (1977).

\section{Callithamnion dasytrichum VER AUTOR}

Taylor (1931), Oliveira-Filho (1977).

\section{Sargassum maximiliani (Shrader) Martius}

Taylor (1931) e Oliveira-Filho (1977).

Caulerpa murrayi Weber van Bosse

Gepp \& Gepp (1905), Taylor (1931) e OliveiraFilho (1977).

AGRADECIMENTOS: A Dra. Mutue Toyota Fujii da Seção de Ficologia do Instituto de Botânica de São Paulo, pela confirmação dos táxons do gênero Laurencia Lamouroux, e valiosas sugestões, ao Prof. Cid José Passos Bastos do Depto. de Botânica, I.B. UFBA, pela rigorosa revisão do manuscrito, ao Dr. Francisco Conde Poyales do Depto. de Biologia Vegetal da Universidade de Málaga e Dr. Michael J. Wynne do Depto. de Biologia da Universidade de Michigan pela revisão criteriosa e sugestões indispensáveis.

\section{REFERÊNCIAS BIBLIOGRÁFICAS}

ALTAMIRANO, M. \& J.M. de CASTRO NUNES, 1997- Contribuciones al macrofitobentos del municipio de Camaçari (Bahia, Brasil). Acta Bot. Malacitana 22: 211-215.

BRAGA, M.R. de A. -1986. Estudos taxonômicos em Gigartina teedii (Roth) Lamouroux e $G$. elegans Greville in Saint-Hilaire (Rhodophyta, Gigartinales). Revta brasil. Bot. 9: 191-199.

BUYS, S. C. \& M.T.M. SZÉCHY, de. -1997Corallina panizzoi Schnetter et Richter (Corallinales, Rhodophyta) en el litoral brasilero. Revta. brasil. Biol., 57 (1): 71-77.

CHOU, R. C-Y. - 1945. Pacific species of Galaxaura. 1. Asexual types. Pap. Mich. Acad. Sci. 30: 3555.

DE TONI, G.B. -1889- Sylloge algarum omnium hucusque cognitarum. I (Sylloge Chlorophycearum): 1-12 + I-CXXXIX +1-1315.

DICKIE, G. -1874- Enumeratio of algae collected from Bahia by H. N. Moseley, M. A., naturalist to H. M. S. "Challenger". J. Linn. Soc. (Bot.) 14: 377 .

GEPP, A. \& E. S. GEPP - 1905- Atlantic algae of the "Scotia". J.Bot., London, 43:109-110.

GOUVEA, E. P. \& Y.M.R. LEITE -1980- A carcinofauna do fital de Halimeda opuntia (Linnaeus) Lamouroux e a variação sazonal de sua densidade. Ciência e Cultura 32 (5): 596600.

GUIMARÃES, S. M. P. B. -1996- Taxonomy of the flattened Solieriaceae (Rhodophyta) in Brazil: Agardhiella and Meristiella. J. Phycol. 32: 656668.

HOWE, M. A. -1928- Notes on some marine algae from Brazil and Barbados. J. Wash. Acad. Sci., Washington 18 (7): 186-194.

HOWE, M. A. \& W.R. TAYLOR -1931- Notes on some new or little-known marine algal from Brazil. Brittonia 1: 7-33.

JOLY, A. B. \& UGADIM, Y. -1966- The reproduction of Ochtodes secundiramea (Montagne) Howe, (GigartinalesRhizophyllidaceae). Bolm. Inst. Oceanogr., São Paulo 15 (1): 55-64.

JOLY, A. B., M. SAZIMA \& J. SEMIR -1976Brazilian deep water marine algae additions to the Brazilian flora IV. Bol. Botânica, Univ. S. Paulo 4: 121-128.

JOLY, A. B., J. SEMIR \& Y. WAKABARA -1978Brazilian deep-water marine algae: addtions to the Brazilian flora-V. Revta. Brasil. Bot. 1: 125-129.

JOLY, A. B., E.C. de OLIVEIRA FILHO \& W. NARCHI -1969a- Projeto de criação de um Parque Nacional Marinho na Região de Abrolhos, Bahia. An. Acad. brasil. Ciênc. (supl.) 41: 247-251. 
JOLY, A. B., M. CORDEIRO-MARINO, N. YAMAGUISHI-TOMITA, Y. UGADIM, E. C. de OLIVEIRA-FILHO \& M.M. FERREIRA 1965a-Additions to the marine flora of Brazil V. Arq. Est. Biol. Mar. Univ. Ceará 5 (1): 6578.

JOLY, A.B., M. CORDEIRO-MARINO, Y. UGADIM, N. YAMAGUISHI-TOMITA \& F.C. PINHEIRO -1965b- New marine algae from Brazil. . Arq. Est. Biol. Mar. Univ. Ceará 5 (2): 79-92.

JOLY, A. B., Y. UGADIM, J.J.G. DIAZ, N. YAMAGUISHI-TOMITA, M. CORDEIROMARINO \& Y. YONESHIGUE-BRAGA 1969b-Additions to the marine flora of Brazil X. Rickia 4: 61-74.

JOLY, A.B., Y. UGADIM, E.C. de OLIVEIRAFILHO \& M. CORDEIRO-MARINO -1967Additions to the marine flora of Brazil VI. Bolm. Fac. Filos. Ciênc. Univ. S. Paulo. 305 (bot.) (2): 171-194.

KANAGAWA, A.I. -1984- Clorofíceas marinhas bentônicas do Estado da Paraíba, Brasil. Tese Doutorado, Universidade de São Paulo, São Paulo. 470p.

LEITE, Y.M.R. -1982- Estudo das algas bentônicas e dos mangues da Baía de Aratu e do estuário de Maragojipe. In: Bahia. Secretaria da Indústria e Comércio (ed). Diagnose ecológica da Baía de Aratu. (Salvador, BA) 3: 165-184.

MARTENS, G. von. -1870- Conspectus algarum Brasiliae hactenus detectarum. Vidensk. Meddr dansk naturh. Foren 2: 297-314.

MARTINS, D.V., M. CORDEIRO-MARINO, N.B. BOCCANERA \& J.M. de CASTRO NUNES, 1991- Clorofíceas marinhas bentônicas do município de Salvador, Bahia, Brasil. Hoehnia 18 (2): 115-133 + 33 il.

MARTIUS, K.F.P. von, F.G. ESCHWEILLER \& C.G.N. von. ESENBECK -1833-Flora Brasiliensis seu enumeratio plantarum in Brasília ... 1, 1: IV + 390pp. (Algae 1-50). 8 vo. Stuttgart et Tübingen.

MÖBIUS, M. -1889- Bearbeirung der von H. Shenk in Brasilien gesammelten Algen. Hedwigia 28: 309-347 + pls. 10-11.

MÖBIUS, M. -1890- Algae brasiliensis a cl. Dr. Glaziou colectae. Notarisia 5: 1065-1090+1 pl.

MONTAGNE, J.F.C. -1839- Cryptogamae brasiliensis seu plantae cellulares quas in itinere per brasiliam...(algae). Annls. Sci. nat. Bot. 12: 42-44.

MOURA, C. W. do N. -1994- Coralináceas com genículo (Corallinales, Rhodophyta) do litoral oriental do Estado do Rio Grande do Norte, Brasil. Dissertação de Mestrado, Universidade Federal Rural de Pernambuco, Recife. 254p.

NUNES, J.M. de C. -1997a- Hypneaceae, Rhodymeniaceae, Ceramiaceae e Rhodomelaceae (Rhodophyta) das praias de Placafor e Itapoã, Município de Salvador, Bahia, Brasil. Biotemas 10 (2): 61-75.

NUNES, J.M. de C. -1997b- Rodofíceas marinhas bentônicas da orla oceânica de Salvador, Estado da Bahia, Brasil. Ínsula. (in press).

OLIVEIRA, E.C. -1989. Uma antiga coleção de algas marinhas do litoral brasileiro. Ciência e Cultura 41 (4): 402-403.

OLIVEIRA-FILHO, E.C. de. -1974- An annotated list of the Brazilian seaweeds in Dickie's herbarium. J. Linn. Soc. (bot.) 69 (3): 229-238.

OLIVEIRA FILHO, E.C. de. -1977-Algas Marinhas Bentônicas do Brasil. São Paulo, 407p. Tese (Livre-Docência) - Instituto de Biociências, Universidade de São Paulo.

OLIVEIRA FILHO, E.C. de \& Y. UGADIM - 1974New references of benthic marine algae to the Brazilian Flora. Bolm. Botânica, Univ. S. Paulo 2: 71-91.

OLIVEIRA FILHO, E.C. de, Y. UGADIM \& E.J. de. PAULA -1979- Comunidades associadas a plantas de Sargassum flutuantes em águas da corrente do Brasil - considerações biogeográficas. Bol. Botânica, Univ. S. Paulo 7: 5-9.

PICCONE, A. -1885- Notizie preliminari intorno alle alghe della "Vettor Pisani" ... Nuovo G. Bot. ital. 17: 185-188.

PICCONE, A. -1886a- Alghe del viaggio di circumnavigazione della "Vettor Pisani". Contr. Phycol. Extra-Ital.: 283-287.

PICCONE, A. -1886b- Alghe del viaggio di circumnavigazione della "Vettor Pisani". Contr. Phycol. Extra-Ital.: 87p. +2 pls.

PICCONE, A. -1889- Nuove alghe del viaggio di circumnavigazione della "Vettor Pisani". Atti Acad. Lincei. Mem. Cl. Sci. Fisiche, Mat. e Nat. 6: 10-63.

PINHEIRO-JOVENTINO, F. -1977- Morfologia, taxonomia e distribuição de Cryptonemia J. 
Agardh, no Brasil (Rhodophyta, Cryptonemiaceae). Arq. Ciênc. Mar 17 (1): 119.

SANTOS, G.V. dos. -1992-Composição e Microdistribuição de comunidades de algas bentônicas no recife de coral da praia de Coroa (Ilha de Itaparica-Bahia-Brasil). Dissertação de Mestrado, Universidade Federal Rural de Pernambuco. 170p.

SEMIR, J. -19977- Aspectos da morfologia e biologia de Colpomenia sinuosa (Scitosiphonaceae, Phaeophyta), na região de Ubatuba. São Paulo, 102p., Dissertação (Mestrado em Ciências) - Instituto de Biociências da Universidade do Estado de São Paulo,

SILVA, P.C., E.G. MENEZ \& R.L. MOE -1987Catalog of the marine algae of the Philippines. Smithsonian Contributions to the Marine Sciences 27. 179p.

SILVA, P.C., P.W. BASSON \& R.L. MOE -1996Catalogue of the benthic marine algae of the Indian Ocean. University of California Press, Berkeley, USA. 1259p.

TAYLOR, W.R. -1930- Algae collected on the Hassler, Albatross and Schmidth Expeditions: I. Marine algae from Brazil. Am. J. Bot. 16: 621-630.

TAYLOR, W.R. -1931- A synopsis of the marine algae from Brazil. Rev. Algol. 5: 279-313.

TEIXEIRA, V.L., R.C. PEREIRA, J.A. MUNIZ \& L.F.F. da SILVA - 1985- Contribuição ao estudo de algas de profundidade da costa sudeste do Brasil. Ciência e Cultura 37 (5): 809-815.

UGADIM, Y. -1987- Distribuição das espécies de Gelidium e Pterocladia (Gelidiaceae Rhodophyta) no litoral brasileiro. Nerítica 2 (supl.): 65-74.

WEBER van BOSSE, A. -1898. Monographie des Caulerpes. Ann. Jard. Bot. Buiteriz 15: 243401.

WILLIAMS, L.G. \& H.L. BLOMQUIST -1947- A collection of marine algae from Brazil. Bull. of the Torrey Botanical Club. 74 (5): 383-397.
WYNNE, J.M. -1998- A checklist of marine algae of tropical and subtropical western Atlantic: first revision. Nova Hedwigia 116:1-155.

YAMAGUISHI-TOMITA, N. -1970- Bolbocoleon jolyi, a new species of Chaetophoraceae (Chlorophyceae) from Redonda Island, Abrolhos, eastern Brazil. Phycologia 9 (2): 125-132.

Aceptado para su publicación en Junio de 1998

Endereço do autor. Departamento de Botânica, Instituto de Biologia da Universidade Federal da Bahia, Campus de Ondina. CEP40.170-210, Salvador, Bahia, Brasil. Departamento de Ciências da Universidade do Estado da Bahia, Rodovia Alagoinhas/Salvador, km 03, CEP48.100-000, Alagoinhas, Bahia, Brasil.

E-mail: jmcnunes@ufba.br 